\title{
La exactitud como obstáculo epistemológico
}

\section{Rigoberto Martínez Escárcega \\ Director del Instituto de Pedagogía Crítica Profesor investigador Centro de Investigación y Docencia}

\section{Resumen}

$\mathrm{E}$

n el presente escrito se desarrolla una

serie de argumentos apegados a la his-

toria de la ciencia, para defender la tesis según la cual un conocimiento exacto no siempre está fundamentado en una verdad, y de cómo un conocimiento verdadero no siempre tiene como origen una medición exacta. Se exponen las diferencias epistemológicas entre un instrumento y una herramienta de investigación. Se muestra cómo un instrumento científico es la "encarnación" de una teoría. Al final se desarrolla una pequeña reflexión sobre cómo las tesis planteadas pueden ayudar a la vigilancia epistemológica en la investigación educativa.
El problema de la gravitación me convirtió en un racionalista creyente, es decir, en alguien que busca la única fuente confiable de la verdad en la simplicidad matemática.

Albert Einstein

No todo conocimiento empíricamente exacto es verdadero, y no todo conocimiento verdadero es empíricamente exacto. Es importante señalar que la exactitud puede llegar a convertirse en un obstáculo del conocimiento científico. Es importante tener en cuenta cómo la astronomía babilónica, la cosmología griega, la teoría de los ecuantes de Ptolomeo y el sistema de Tycho Brahe persiguieron con denuedo la exactitud empírica, hasta lograr con el trascurrir de los siglos una alta capacidad predictiva -se podían medir los años solares, los meses lunares, los solsticios y los equinoccios con mucha precisión- $y$, sin embargo, caminaron detrás de un supuesto 
completamente falso, que la Tierra era el centro del universo. En contraparte, la teoría de Copérnico, aunque matemáticamente económica, arrojaba una gran cantidad de lagunas y de inconsistencias empíricas, pero partía de un supuesto verdadero: los tres movimientos de la Tierra, el de rotación, el de traslación y el de la inclinación sobre su eje. El conocimiento exacto no es un síntoma de lo verdadero, y el conocimiento verdadero no siempre tiene como remate un dato empíricamente exacto.

\section{II}

Vamos a tratar de argumentar estas tesis con un ejemplo en el terreno de la ciencia física. Analicemos la primera ley con validez universal establecida por parte de Galileo Galilei. Como sabemos hoy en día, gracias a los estudios históricos de Alexandre Koyré (2005), Galileo estableció que la aceleración de los cuerpos en caída libre, guarda una proporcionalidad entre la distancia recorrida y el cuadrado del tiempo. Este genial descubrimiento fue producto más de una abstracción matemática que de una simple observación empírica. Para demostrar esta afirmación tenemos los terribles errores que cometió Galileo al momento de aplicar la ley de la aceleración al movimiento de objetos reales.

Galileo sustituyó la caída libre de los cuerpos por el plano inclinado y el péndulo, una nueva situación experimental que proporciona más oportunidad para medir el tiempo de caída de los cuerpos. Además de la constante universal de la aceleración, estableció que en un plano inclinado la velocidad de la caída es proporcional al seno del ángulo de inclinación y que, en el péndulo, la longitud de los hilos de los cuales cuelgan los objetos es directamente proporcional al cuadrado de los tiempos de oscilación.

Años después de la publicación de los descubrimientos de Galileo, el experimentador Marin Mersenne los sometió a la verificación empírica. Los resultados obtenidos por Mersenne, repetidos más de cincuenta veces, demuestran que un cuerpo pesado no atravesará en cinco segundos 100 codos como lo reporta Galileo, sino 180 codos. Los resultados de Mersenne suponen una diferencia de un 80 por 100 de los publicados por Galileo; concluyen que un cuerpo al caer atraviesa 3 pies en medio segundo, 12 en un segundo, 48 en dos, 108 en tres y 147 en tres y medio segundos. Las mediciones empíricas de Mersenne demuestran la inexactitud de los datos hechos públicos por Galileo, donde el margen de error es imperdonable, científicamente hablando. A pesar de la inexactitud de los datos que reporta Galileo Galilei, los resultados de los experimentos de Marin Mersenne demuestran de forma contundente que la ley de la aceleración de los cuerpos en caída libre es verdadera. $\mathrm{He}$ aquí el ejemplo de un conocimiento verdadero pero empíricamente incorrecto.

Las leyes de la aceleración están en contradicción con los resultados empíricos obtenidos por Galileo. Lo paradójico del asunto, es que los errores experimentales de Galileo en vez de hacerlo un científico despreciable, lo elevan a la categoría de un pensador genial; demuestran que al formular las leyes de la 
aceleración de los cuerpos en caída libre, no estaba supeditado a los datos empíricos de la observación ingenua, sino que fueron la base para generar una ruptura epistemológica. $\mathrm{Al}$ construir un conocimiento científico, Galileo empleó de forma principal la abstracción y la reflexión matemática, más que la simple observación. Lo verdaderamente enigmático del pensamiento de Galileo es que al formular las leyes de la aceleración, pensó en el movimiento de los cuerpos en el vacío, donde toda aceleración es uniformemente desuniforme, independiente del peso y la masa. El resultado de tal hazaña es un conocimiento verdadero en el terreno teórico, pero impreciso en su comprobación empírica.

\section{III}

Ahora veamos el caso contrario, un conocimiento empíricamente exacto, pero científicamente falso.

Galileo Galilei establece que dos cuerpos en caída libre tienen la misma aceleración, independientemente de su peso. Tal afirmación tiene su confirmación exacta en el vacío. Este supuesto contradice toda la física aristotélica que predominó durante siglos, la cual sostenía que existe una relación directa entre el peso de los cuerpos y su tiempo en caída libre. Galileo se acercó a la demostración de su teoría al experimentar con el plano inclinado y el péndulo. Así, encontró que dos cuerpos de diferente peso en un péndulo, sostenidos por hilos de igual longitud, tenían tiempos iguales de oscilación. Además demostró que la duración del periodo de oscilación en el péndulo, era semejante en dos cuerpos con diferente ángulo de oscilación. De ahí dedujo la relación directamente proporcional entre el periodo de oscilación y la raíz cuadrada de la longitud del péndulo. A cualquier científico le hubieran bastado estos descubrimientos para detenerse en su trabajo intelectual, pero no a Galileo que se propuso desafiar el pensamiento aristotélico que había dominado durante más de un milenio. Galileo comparó el movimiento pendular con el movimiento de los cuerpos en caída libre, y observó que el movimiento pendular era más rápido. Por tanto, estableció que la caída a lo largo del arco era la más rápida posible y, que, por ende, el movimiento del péndulo era isócrono. A pesar de la elegancia con que plantea Galileo sus hallazgos y su magistral intuición matemática, el movimiento isócrono del péndulo es falso.

La falsedad del movimiento isócrono del péndulo, se hizo evidente al momento de tratar de aplicar el movimiento pendular a la construcción de un cronómetro exacto.

El primero que intentó utilizar el movimiento pendular para medir el tiempo, según parece, fue Mersenne en 1636. Después de comprobar de forma empírica la ley de la aceleración de los cuerpos en caída libre, Mersenne se propone comprobar el movimiento isócrono del péndulo, con la construcción de un péndulo cuyo periodo de oscilación sea exactamente a un segundo. A pesar de sus mediciones escrupulosamente exactas y la repetición por decenas de veces de sus experimentos, los resultados fueron infructuosos. Al final de un vigoroso trabajo, hubo de aceptar 
que sus experimentos eran un completo fracaso o que la teoría era falsa. Años después, en 1644, descubre que el movimiento pendular no es isócrono.

Al mismo tiempo que Mersenne realizaba sus experimentos, un grupo de sabios jesuitas encabezados por Giambattista Riccioli, en Italia, llevaban a cabo una serie de experimentos para comprobar empíricamente la ley de la caída libre de los cuerpos y determinar su valor empírico.

Riccioli no admite nada como evidente. Como punto de partida, trata de comprobar si la longitud del péndulo y su periodo de oscilación se relacionan tal y como sostiene Galileo. También trata de confirmar el isocronismo de las oscilaciones pendulares. Y por último, trata de determinar el periodo de un péndulo para obtener un instrumento preciso de medición. Empieza por construir un péndulo sencillo y comprobar la igualdad de los periodos de oscilación de dos cuerpos de diferente peso. Utilizó una clepsidra romana para medir el tiempo. Posteriormente, confirmó el axioma de Galileo sobre la relación entre la longitud del péndulo y su periodo de oscilación. Riccioli se da cuenta de la importancia del descubrimiento de Galileo, ya que el isocronismo del péndulo podría ser utilizado para construir un cronómetro preciso. Entonces, le quedó claro que para poder utilizar el péndulo como un instrumento preciso de medición del tiempo, era necesario determinar con anterioridad el valor exacto de su periodo, tarea a la que se consagró con pasión.
Para empezar, construyó un péndulo y midió sus periodos de oscilación con una clepsidra, dando resultados satisfactorios: 900 oscilaciones en un cuarto de hora, tal y como había previsto la teoría. Riccioli procedió entonces a verificar tales resultados a través de la medición de un cuadrante solar. Durante seis horas seguidas contó las oscilaciones con un resultado desastroso: 21,706 oscilaciones en vez de 21,660 previstas. Preparó otro péndulo $\mathrm{y}$, con ayuda de nueve padres jesuitas, volvió a contar durante veinticuatro horas consecutivas; se obtuvo como resultado 87,998 oscilaciones, mientras que el día solar sólo contiene 86,640 segundos. Riccioli construyó otro péndulo y para aumentar la precisión decide tomar como unidad de medida la duración del día sideral. Se comenzó a contar a partir del 12 y hasta el 13 de mayo del 1642. Nuevo fracaso: 86,999 oscilaciones en lugar de las 86,400 previstas. Sin rendirse aún, Riccioli decidió hacer un nuevo experimento y construyó otro péndulo. El conteo se realizó en tres ocasiones durante tres noches, el 19 y el 28 de mayo y el 2 de junio de 1645. Dos veces las cantidades son de 3,212 y, la tercera de 3,214 oscilaciones, para los 3,192 segundos previstos. Llegado a este punto, Riccioli sacó la conclusión de que a través de métodos directos nunca llegaría a la construcción de un péndulo cuyo periodo de oscilación fuera exactamente igual a un segundo. Así que sólo estableció dicho péndulo por métodos indirectos, utilizando tablas de equivalencias.

El mérito de construir un reloj exacto pertenecerá a Huygens, quien realiza tal hazaña 
en 1659, al aplicar una nueva teoría matemática al movimiento pendular, y no buscando meticulosamente la exactitud de los datos empíricos. Huygens demostró que la caída de los cuerpos a lo largo del arco no es la más rápida posible y que, por tanto, el movimiento pendular no es isócrono; pudo ver que la cicloide es a la que le corresponde un movimiento isócrono. Huygens mostró que los métodos indirectos llevados a cabo a través de la teoría son los más indicados para construir hechos científicos. El análisis de este ejemplo nos plantea cómo un conocimiento empíricamente exacto puede estar fincado en un conocimiento falso. Las mediciones de Mersenne y de Riccioli eran muy rigurosas y exactas, pero estaban basadas en un error teórico, en el isocronismo del movimiento pendular. Para construir hechos científicamente fructíferos es necesario revolucionar la teoría. "En la investigación científica, el enfoque directo no es el mejor ni el más fácil; los hechos empíricos no pueden alcanzarse sin recurrir a la teoría" (Koyré, 2000: 295).

\section{IV}

El ejemplo de Huygens y la invención del reloj dejan algunos aprendizajes importantes para seguir desarrollando un psicoanálisis del conocimiento objetivo, en los términos en que lo hiciera Gastón Bachelard (2007). Huygens triunfa ahí donde habían fracasado sus predecesores, logra ver un objeto invisible para una epistemología empirista. Gracias al desplazamiento epistemológico generado por una nueva teoría, logra identificar el error teórico en el que se habían basado los experimentos de Mersenne y de Riccioli. Es también gracias a su mérito teórico que se exime de realizar una medición empírica exacta. Huygens logra determinar el periodo de oscilación de la cicloide, perfectamente isócrono, igual a un segundo, sin necesidad de experimentar con péndulos, sino basándose en una nueva interpretación geométrica del problema.

El episodio de Mersenne, Riccioli y Huygens ilustra, de nueva cuenta, un falso problema científico planteado desde una problemática no científica. Basta mutar de problemática y lo que parece una respuesta se convierte en una pregunta de investigación, el problema se convierte en una interrogante científica. La problemática teórica es lo que posibilita la delimitación científica de un problema de investigación. Por lo que, tanto el objeto como el problema que lo hace posible, están determinados por la problemática teórica desde la cual son formulados. "Un objeto de investigación, por más parcial y parcelario que sea, no puede ser definido y construido sino en función de una problemática teórica que permita someter a un sistemático examen todos los aspectos de la realidad puestos en relación por los problemas que le son planteados" (Bourdieu, 1996: 54).

El reloj de Huygens y el telescopio de Galileo, son perfectos ejemplos de una teoría científica aplicada. Como lo describe Alexandre Koyré: “... no sólo los experimentos válidos se fundan en una teoría, sino que también los medios que permiten realizarlos no son otra cosa que teoría encarnada" (2000: 300). 
Una teoría científica bien delimitada permite acercarse a un conocimiento empírico exacto. Es decir, que la exactitud sólo es posible lograrla con la teoría. La exactitud empírica tiene como condición una teoría verdadera, pero una teoría verdadera no tiene siempre su correlato en una medición empírica exacta.

Además de reflexionar sobre las diferencias entre la mediación y la verdad, es importante delimitar la diferencia epistemológica entre una herramienta y un instrumento de investigación. Las herramientas son la extensión de los sentidos, datos empíricos acumulados de forma artesanal de generación en generación. La herramienta nos ayuda a observar el mundo sensible. En cambio, un instrumento de investigación, es la encarnación de una teoría, permite ver los objetos invisibles para el sentido común. Los instrumentos de investigación como encarnación de la teoría, no sólo permiten interactuar con el mundo, sino conocer facetas desconocidas de lo real. Los instrumentos de investigación son producto de una revolución científica, de una mutación de problemática, mientras que las herramientas son el resultado de una simple acumulación de datos empíricos. "El instrumento de medida siempre termina por ser una teoría, y ha de comprenderse que el microscopio es una prolongación del espíritu más que del ojo" (Bachelard, 2007: 285). En este mismo sentido, Alexandre Koyré, señala la construcción de instrumentos de investigación como encarnaciones de la teoría:

Galileo es también el que, por lo menos en mi opinión, construyó o creó el pri- mer verdadero instrumento científico. Ya he dicho que los instrumentos de observación de Tucho Brahe eran ya de una precisión desconocida hasta entonces, pero los instrumentos de Tycho Brahe, como todos los instrumentos de la astronomía anteriores a Galileo, eran instrumentos de observación; eran a lo sumo, instrumentos de medida -más precisos que los de sus predecesoresde hechos simplemente observados. En cierto sentido, aún son herramientas, mientras que los instrumentos galileanos -y esta es aplicable tanto al péndulo como al telescopio- son instrumentos en el más fuerte sentido del término: son encarnaciones de la teoría. El telescopio galileano no es un simple perfeccionamiento del anteojo "bátavo»; está construido a partir de una teoría óptica; está construido con una cierta finalidad científica, a saber, para revelar a nuestros ojos las cosas que son invisibles a simple vista. Tenemos aquí el primer ejemplo de una teoría encarnada en la materia, que nos permite franquear los límites de lo observable en el sentido de lo que se ofrece a la percepción sensible, fundamento experimental de la ciencia pregalileana (Koyré, 2000: 50).

Una buena medición se obtiene gracias a la aparición de un instrumento científico y un instrumento científico se construye como base de la encarnación de una teoría científica. Sin teoría científica no hay instrumento 
científico y sin instrumento científico sólo tenemos datos empíricos producto de la observación ingenua. Los buenos instrumentos científicos no se construyen con base en los datos empíricos, sino como encarnación de una mutación de problemática teórica. La ciencia es fundamentalmente un esfuerzo teórico. "Creo profundamente que la ciencia es esencialmente teoría, y no recolección de hechos" (Koyré, 2000: 152).

Un conocimiento científico no se construye al realizar mediciones empíricas exactas o al recolectar de forma meticulosa información empírica, sino mediante un esfuerzo teórico, un cambio de terreno, un desplazamiento epistemológico capaz de generar una ruptura con el conocimiento de sentido común.

\section{V}

La verdad no siempre surge de un conocimiento exacto, y un conocimiento exacto no siempre está asentado sobre una verdad. El desarrollo y argumentación de estas tesis en el campo de la física podrían dejar algunas enseñanzas a la investigación educativa.

Lo primero que hay que señalar es que la medición rigurosa del dato no es un criterio de cientificidad; el dato debe ser interrogado y cuestionado por una teoría para acotar las prenociones inconscientes del sentido común. A partir de la teoría se debe construir el dato. El camino inductivo que parte del dato a la teoría da forma a un realismo ingenuo, posicionamiento epistemológico hegemónico en la investigación educativa. El camino deductivo que va de la teoría al dato da forma a una postura racionalista. Si sólo se persigue que el dato ratifique a la teoría se corre el peligro de caer en un racionalismo dogmático. Es necesaria una teoría que genere datos, que se rebele a la contemplación y se fundamente en la acción. El dato se construye no se contempla. La ciencia debe ser generativa no contemplativa. Una teoría científica no sólo predice fenómenos empíricos, genera hechos científicos. Es necesario el surgimiento de un racionalismo dialéctico.

En segundo lugar, la investigación educativa está atrapada en medio de la falsa disputa entre lo cuantitativo y lo cualitativo, que tanto daño ha hecho a la generación de conocimiento original. Es importante desmitificar al dato empírico, poner atención en la vigilancia epistemológica, independientemente del procedimiento metodológico adoptado en la investigación.

Finalmente habría que señalar la exégesis del método en la investigación educativa. Se elige de forma a priori el método y a posteriori se define el problema de investigación. Es importante señalar cómo el método debe subordinarse al problema de investigación, y el problema de investigación debe supeditarse a la problemática teórica, a partir de la cual se interroga al dato y se leen sus respuestas.

Espero que este pequeño ensayo ayude a reflexionar sobre el importante papel de la teoría en la investigación educativa. 


\section{Bibliografía}

Bachelard, Gaston (1988), El compromiso racionalista, México, Editorial Siglo XXI, 179 pp.

Bachelard, Gaston (2007), La formación del espíritu científico. Contribución a un psicoanálisis del conocimiento objetivo, México, Editorial Siglo XXI, 302 pp.

Bourdieu, Pierre, Jean-Claude Chamboredon y Jean-Claude Passeron (1996), El oficio de sociólogo, México, Editorial Siglo XXI, 372 pp.
Koyré, Alexandre (1992), Del mundo cerrado al universo infinito, México, Editorial Siglo XXI, 268 pp.

Koyré, Alexandre (1994), Pensar la ciencia, México, Edición Paidós, $145 \mathrm{pp}$.

Koyré, Alexandre (2000), Estudios de historia del pensamiento científico, México, Editorial Siglo XXI, 394 pp.

Koyré, Alexandre (2005), Estudios galileanos, México, Editorial Siglo XXI, $332 \mathrm{pp}$. 